\title{
GESTATIONAL DIABETES MELLITUS MAY ASSOCIATE WITH ATHEROSCLEROSIS DUE TO HIGH MEAN PLATELET VOLUME WHICH IS A DETERMINED OF PLATELET FUNCTION
}

\section{Dr.Senay Arikan Durmaz¹, Dr.Ayse Carlioglu², Dr.Bilge Eris', Dr.Faruk Yıldiz², Dr.Halime Kılıç³ \\ 1Department of Endocrinology and Metabolism, Kirikkale University, Kirikkale, Turkey \\ 2Department of Endocrinology and Metabolism, Erzurum Region Education and Research Hospital, Erzurum, Turkey \\ ${ }^{3}$ Department of Gynecology \&Obstetrics, Nenehatun Doğumevi Hospital, Erzurum, Turkey}

\section{Introduction:}

Gestational Diabetes Mellitus (GDM) is defined as a disorder of carbohydrate metabolism arising during pregnancy and this metabolism is negatively affected by the natural course of pregnancy. The increased concentration of cortisone, prolactin, human placental lactogen and leptin in the circulation play an important role in the development of insulin resistance in GDM.3$5 \%$ of all pregnancies are negatively affected by gestational diabetes mellitus. Women diagnosed with GDM represent approximately $87 \%$ of all cases of diabetes complicating pregnancy.

Placental dysfunction is assumed to be partly accountable for high frequency in diabetic women. Diabetic placentea transfers more arachidonic acid to prostanoids. Thus, higher Thromboxane A2 (TxA2) and lower Prostoglandin $\mathrm{I}_{2}(\mathrm{PGI} 2)$ on both the fetal and maternal side of the circulation create hypercoagulable conditions. Larger platelets with a higher mean platelet volume (MPV) produce higher amounts of the prothrombotic factor. Furthermore, the hypercoagulable conditions stimulated by GDM could be correlated to the variations in TxA2 and/or PGI2 production.

Aim:

Mean platelet volume (MPV), which is a determinant of platelet function, is an independent risk factor for atherosclerosis. The aim of this study is to evaluate MPV values in GDM.

\section{Material and methods:}

Forty-nine women with gestational diabetes mellitus (mean age 28.24 .7 years age) and 40 healthy pregnant women (mean 26.24 .6 years age) who came to our out-patient clinic of Endocrinology were included in the study. GDM group and the control group were matched for age. All the study subjects were evaluated by platelet parameters. All complete blood count analysis was performed with an automatic hematologic analyzer.

\section{Results:}

The MPV levels in GDM were significantly higher than controls (7.9 0.6 and $7.60 .54 \mathrm{fL} ; \mathrm{p}=0.0001$, respectively). In addition, there was found a positive correlation between MPV and fasting glucose $(F G)$ level $(r=0.52 ; p=0,03)$.

The multiple regression analysis of MPV was performed. High MPV value in GDM was independent from age, body mass index and fasting glucose. levels $(\beta=0.238$, $p=0.046$ ).

No statistically significant differences were found for the other parameters such as hemoglobin, platelet count, lymphocyte count, neutrophil count, white blood cell count, platelet distribution width and plateletcrit.

\section{Discussion:}

In this study gestational diabetic pregnancies were compared to normal pregnancies population. The patients with GDM were observed to have a higher MPV. Platelet volume is a marker of platelet activation and function and measured using MPV. In previous study MPV was observed to be higher gestational diabetics as we found.

\section{Conclusion:}

Our findings suggest that GDM are susceptible to increased MPV values which contribute to increased risk of atherosclerosis. Future prospective studies that MPV was compared with other risk factors of atherosclerosis are required to determine their clinically importance in GDM. 